\title{
A COMPARISON OF TRAFFIC SIGN COMPREHENSION USING STATIC, DYNAMIC AND INTERACTIVE PRESENTATION MEDIA
}

\author{
Susan T. Chrysler, James Wright, and Alicia Williams \\ Center for Transportation Safety \\ Texas Transportation Institute \\ 308 Gilchrist Building \\ 3135 TAMU \\ College Station, TX 77843-3135 \\ E-mail: s-chrysler@tamu.edu
}

\begin{abstract}
Summary: Traditionally, traffic sign comprehension has been tested using paper-and-pencil tests with line drawings of signs and uncontrolled viewing time of the test signs. This study compares these types of tests to dynamic tests using an interactive driving simulator. Multiple-choice tests concerning sign comprehension were administered to five groups of Texas drivers following exposure to traffic sign stimuli via line drawings, still computer drawings in a roadway context either with or without controlled exposure, a video of a "drive through" from the simulator, or driving in the simulator itself. Results show interesting differences among the groups that suggest that past studies may have overestimated sign comprehension.
\end{abstract}

Past research assessing traffic sign comprehension has often employed simple paper and pencil tests that ask, "What do you think this sign means?" The test signs have been typically shown in isolation as black-and-white line drawings, still photographs, or 35mm slides. Often these presentation media allow unlimited viewing time of the test sign. A review of past research on comprehension has shown that these methods may be producing ceiling effects. In several studies, comprehension for all variants of a guide sign exceeded $85 \%$. With such a limited range, differences between experimental sign designs are often not significant. A few studies have tried to impose a viewing time limitation by controlling exposure duration. Others have used video or film of actual roadways to present test signs in a driving context and to limit viewing time. With the advent of interactive driving simulation, a few studies have tested traffic sign comprehension in simulators.

It is not known what effect these different presentation media have on sign comprehension test performance. It is easy to add driving environment context by providing images of signs in appropriate roadway situations. Movement and time pressure can be added by using computer animation or film. Cognitive load can be increased by imposing additional tasks, either by asking the participant to drive in an interactive simulator, or by giving monitoring tasks in a passive video presentation.

The current study provides a direct comparison of all these presentation media. A betweensubjects design was used with five presentation media groups. An array of traffic signs, including guide, warning, regulatory, and work zone signing, was pulled from previous research. The test of comprehension was mainly a multiple-choice test, but included some behavior-based dependent variables for the driving simulator condition. The presentation media varied along continuums of driving context and dynamism. 
- Paper and pencil test with simple line drawings of signs with no roadway context. This test was self-administered.

- Paper and pencil test with black-and-white screen dumps from the driving simulator showing each target sign in a roadway context. This test was self-administered.

- Computer slide presentation with color graphics of signs shown in a roadway scene derived from screen dumps from the driving simulator. This test used an exposure fixed at five seconds.

- Video loop of the driving environment generated by driving simulator software presented to a group in a classroom. The test questions were answered on paper after the sign had been passed and while the video was paused.

- Full immersion presentation of the same driving environment with the subject driving in wrap-around simulator. The test questions were answered on paper after the sign had been passed and the drivers had pulled to the side of the road. In addition to the multiplechoice questions, driver behavior as evidenced by turn signal activation and lane changes was recorded.

The results show distinct differences between the presentation media. For signs with fine detail and small text, the video and driving simulator conditions produced more incorrect responses than the paper-and-pencil tests. For lane drop signs, a few participants in the driving simulator condition made the appropriate lane change maneuver, but answered incorrectly on the multiplechoice question about the sign's meaning. This is the type of result that argues for driving-based methods of sign comprehension. Drivers may not overtly be able to explain, or even recognize in a multiple-choice test, the meaning of a sign, but their driving performance exhibits some level of comprehension.

There are downsides to driving simulator-based tests. The time and expense to build and maintain a simulator are obvious barriers to entry. In addition, we found extremely high levels of simulator sickness with our participants over the age of 55, particularly with older women. Seventy-five percent of our older drivers had to stop the experiment because of discomfort and motion sickness. The decision was made to cease data collection for the older driver group. The majority of participants who did get sick asked to stop within the practice session. Most of the others asked to stop shortly after the first pull-over. We believe that the act of pulling over and stopping caused some discomfort because of the virtual movement of the car as it pitched forward when the brakes were applied.

We would like to continue to develop lower-cost methods of testing sign comprehension. One major advantage of a portable method, such as a video dump from a simulator, is the portability for data collection. If comparable performance is achieved for the groups driving in the simulator and the group watching the video, future research could use this less expensive and faster method of comprehension testing. 